\section{Flows and flaws in primary central nervous system lymphoma}

\author{
Andrés J. M. Ferreri, Gerald Illerhaus, Emanuele Zucca and Franco Cavalli \\ on behalf of the International Extranodal Lymphoma Study Group
}

We read with great interest the article by Ansell and Rajkumar (Trials and tribulations in primary CNS lymphoma. Nat. Rev. Clin. Oncol. 7, 125-126; 2010), proposing an algorithm for treatment of primary central nervous system lymphoma (PCNSL). ${ }^{1}$ The authors are to be commended for their effort to construct a therapeutic flow chart in a field where level of evidence is very low. As a natural consequence, some of their recommendations deserve to be better discussed and the use of the proposed algorithm in the everyday practice should be taken into account with caution.

The key decisional intersection in the algorithm proposed by Ansell and Rajkumar is patient eligibility for high-dose chemotherapy supported by autologous stem-cell transplantation (HDC-ASCT). Such an approach is, however, hardly applicable in practice, considering that worldwide experience with this strategy in PCNSL is limited to small retrospective series and a few phase II trials. ${ }^{2}$ This preliminary experience seems to suggest that HDC-ASCT is feasible in young and fit patients, and is associated with encouraging results, mostly if a thiotepacontaining conditioning regimen is used. ${ }^{2}$ However, these observations, as well as the hypothesis that HDC-ASCT is associated with better neurotolerability with respect to consolidation whole-brain irradiation, remain to be addressed in future randomized trials.

Efficacy of primary (induction) chemotherapy has a central role in the outcome of PCNSL, even among patients referred to consolidation HDC-ASCT. In the Ansell and Rajkumar algorithm, ${ }^{1}$ methotrexate at $8 \mathrm{~g} / \mathrm{m}^{2}$ was suggested as induction therapy both for patients eligible for HDC-ASCT and for patients $>60$ years old who were ineligible for HDC-ASCT. However, the superiority of $8 \mathrm{~g} / \mathrm{m}^{2}$ over lower methotrexate doses (that is, $3 \mathrm{~g} / \mathrm{m}^{2}$ ) has not been investigated. Furthermore, trials on HDC-ASCT in PCNSL have shown unsatisfactory activity of methotrexate $8 \mathrm{~g} / \mathrm{m}^{2}$ as induction therapy, with complete remission rates (CRR) of 17-23\%. ${ }^{3,4}$ Among series managed with methotrexate $8 \mathrm{~g} / \mathrm{m}^{2}$ as exclusive treatment, the promising $52 \%$
CRR reported in an American trial ${ }^{5}$ was not confirmed in an analogous German trial, ${ }^{6}$ in which CRR was $29 \%$ and accrual was halted early owing to lack of treatment efficacy. Importantly, methotrexate $8 \mathrm{~g} / \mathrm{m}^{2}$ monochemotherapy has been frequently associated with doselimiting toxic effects and with significant neurotoxicity in surviving patients (the rate of leukoencephalopathy was $58 \%$ at 4 years), ${ }^{6}$ raising important concerns about treatment feasibility. Overall, these observations seem to suggest that methotrexate $8 \mathrm{~g} / \mathrm{m}^{2}$ should be removed from Ansell and Rajkumar's algorithm, and replaced by a methotrexate-cytarabine

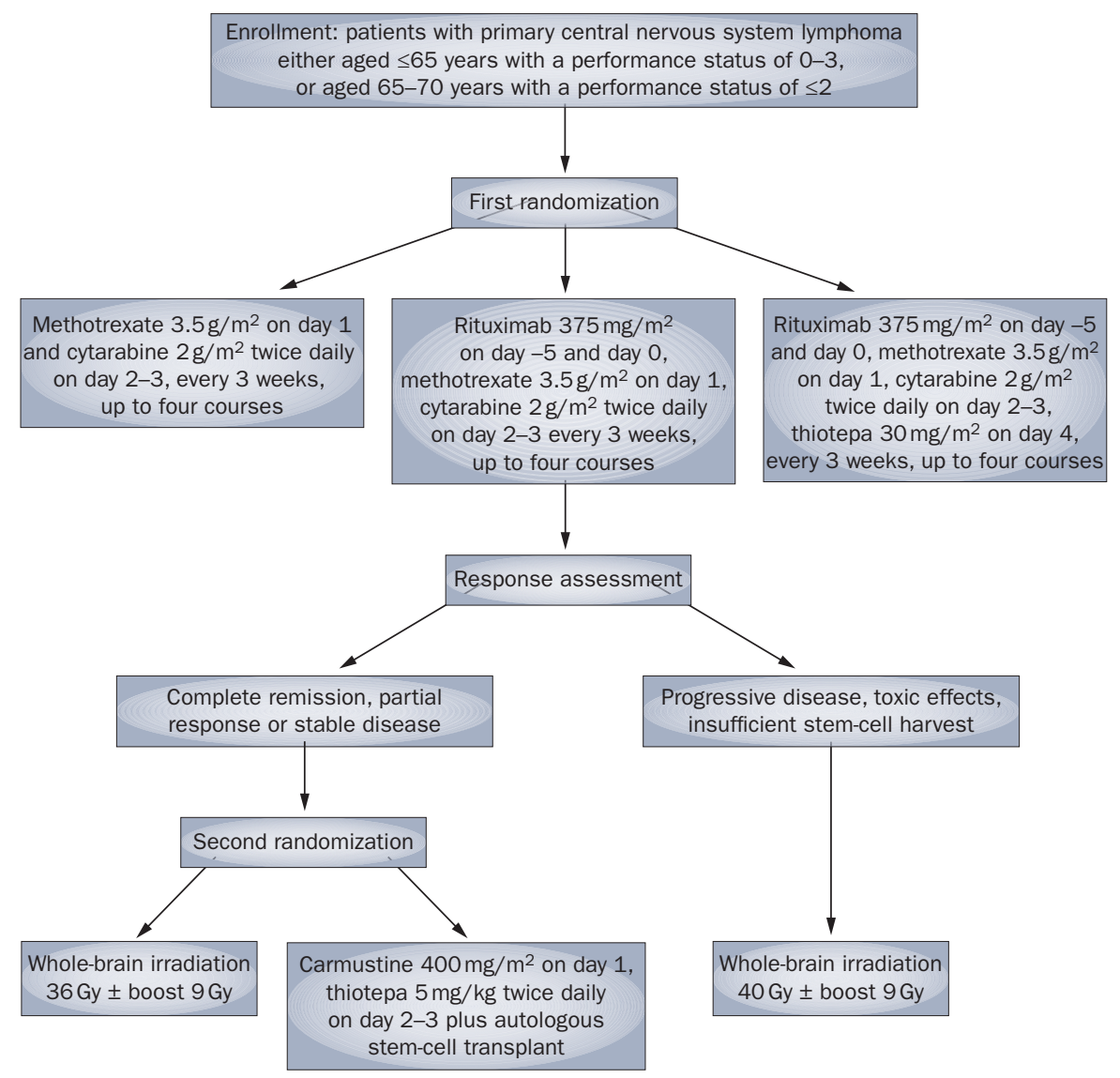

Figure 1 | Flow chart of the IELSG \#32 trial (clinicaltrials.gov: NCT01011920). In the first randomization, three different combinations will be analyzed, using the International Extranodal Lymphoma Study Group risk score ${ }^{10}$ as stratification criterion and complete remission rate as primary end point. Leukapheresis for stem-cell collection will be performed after the second course in the three arms. In the second randomization, two different consolidation therapies will be compared: conventional whole-brain irradiation versus high-dose chemotherapy supported by autologous stem-cell transplantation, using primary chemotherapy regimen and response degree as stratification criteria and 2-year progression-free survival as the primary end point. 
combination, the superiority of which over single-agent methotrexate in patients $\leq 75$ years old has been demonstrated at the highest evidence level in PCNSL. ${ }^{7}$

As noted by Ansell and Rajkumar, clinical trials aimed to identify treatment regimens with higher remission rates should be encouraged. Accordingly, a new international randomized trial is being conducted under the sponsorship of the International Extranodal Lymphoma Study Group (the IELSG \#32 trial). This trial includes a double randomization (Figure 1). First, registered patients will be randomly assigned to receive one of three chemotherapy combinations of methotrexate-cytarabine, rituximab, an anti-CD20 monoclonal antibody that has changed the natural history of diffuse large B-cell lymphoma, ${ }^{8}$ which is the most common lymphoma category arising in the CNS, and thiotepa, an alkylating agent with excellent CNS bioavailability. This stage will explore whether the activity of induction methotrexate-cytarabine combination may be further improved by the addition of rituximab or rituximab and thiotepa. Patients with responsive or stable disease after four chemotherapy courses will be referred to the second randomization to explore whether HDC-ASCT (experimental arm) may provide a more favorable risk:benefit ratio than consolidation whole-brain irradiation (control arm). Importantly, the effect of primary chemotherapy and consolidation strategies on superior neurological functions will be addressed by using the International Primary CNS Lymphoma Collaborative Group panel of neuropsychological tests. ${ }^{9}$

In accordance with the conclusions of Ansell and Rajkumar, we hope that the IELSG \#32 trial could contribute to further optimization of the care of patients with PCNSL. In the meantime, themethotrexate-cytarabine combination followed by whole-brain irradiation ${ }^{7}$ remains the standard treatment in ordinary clinical practice. The use of consolidation HDC-ASCT as an unverified, active option to reduce the putative risk of radiotherapy-related neurotoxicity should be discussed with selected patients.

Unit of Lymphoid Malignancies, Department of Oncology, San Raffaele Scientific Institute, Via Olgettina 60, 20132 Milan, Italy

(A. J. M. Ferreri). Department of Hematology and Oncology, University Medical Center, Hugstetter Street 55, 79106 Freiburg, Germany (G. Illerhaus). Istituto Oncologico della Svizzera Italiana, Ospedale San Giovanni, Viale Officina 3, CH 6500 Bellinzona, Switzerland (E. Zucca, F. Cavalli).

Correspondence to: Andrés J. M. Ferreri andres.ferreri@hsr.it

doi:10.1038/nrclinonc.2010.9-c1

\section{Acknowledgments}

The authors wish to thank Michele Reni (Medial Oncology Unit, San Raffaele Scientific Institute, Milan, Italy) and Jürgen Finke (Department of Hematology and Oncology, University Medical Center, Freiburg, Germany) for their excellent assistance with the manuscript.

Competing interests

The authors declare no competing interests.
1. Ansell, S. M. \& Rajkumar, S. V. Trials and tribulations in primary CNS lymphoma. Nat. Rev. Clin. Oncol. 7, 125-126 (2010).

2. Ferreri, A. J., Crocchiolo, R., Assanelli, A. Govi, S. \& Reni, M. High-dose chemotherapy supported by autologous stem cell transplantation in patients with primary central nervous system lymphoma: facts and opinions. Leuk. Lymphoma 49, 2042-2047 (2008).

3. Illerhaus, G. et al. High-dose chemotherapy and autologous stem-cell transplantation without consolidating radiotherapy as first-line treatment for primary lymphoma of the central nervous system. Haematologica 93, 147-148 (2008).

4. Montemurro, M. et al. Primary central nervous system lymphoma treated with high-dose methotrexate, high-dose busulfan/thiotepa, autologous stem-cell transplantation and response-adapted whole-brain radiotherapy: results of the multicenter Ostdeutsche Studiengruppe Hamato-Onkologie OSHO-53 phase II study. Ann. Oncol. 18, 665-671 (2007).

5. Batchelor, T. et al. Treatment of primary CNS lymphoma with methotrexate and deferred radiotherapy: a report of NABTT 96-07. J. Clin. Oncol. 21, 1044-1049 (2003).

6. Herrlinger, U. et al. NOA-O3 trial of high-dose methotrexate in primary central nervous system lymphoma: final report. Ann. Neurol. 57, 843-847 (2005).

7. Ferreri, A. J. et al. High-dose cytarabine plus high-dose methotrexate versus high-dose methotrexate alone in patients with primary CNS lymphoma: a randomised phase 2 trial. Lancet 374, 1512-1520 (2009).

8. Coiffier, B. State-of-the-art therapeutics: diffuse large B-cell lymphoma. J. Clin. Oncol. 23 6387-6393 (2005).

9. Correa, D. D. et al. Cognitive functions in primary central nervous system lymphoma: literature review and assessment guidelines. Ann. Oncol. 18, 1145-1151 (2007).

10. Ferreri, A. J. et al. Prognostic scoring system for primary CNS Iymphomas: the International Extranodal Lymphoma Study Group experience. J. Clin. Oncol. 21, 266-272 (2003). 